\title{
Mediastinal Lymph Node Biopsy
}

National Cancer Institute

\section{Source}

National Cancer Institute. Mediastinal Lymph Node Biopsy. NCI Thesaurus. Code C137934.

Removal or sampling of lymph nodes in the mediastinum. 